\title{
Web-Based Health Monitoring System and Textual Mining
}

\author{
Md Sabbir ${ }^{1}$, Al Bakin Tushar ${ }^{2}$, Feroz Riazul Iqbal ${ }^{3}$, Sourav Gupta Ananda ${ }^{4}$, Naima Hassan ${ }^{5}$, \\ Sifat Rahman Ahona ${ }^{6}$ \\ ${ }^{1}$ American International University-Bangladesh, Bangladesh, 17-34852-2@ student.aiub.edu \\ ${ }^{2}$ American International University-Bangladesh, Bangladesh, 17-35626-3@ student.aiub.edu \\ ${ }_{3}^{3}$ American International University-Bangladesh, Bangladesh, 17-34333-1@ student.aiub.edu \\ ${ }^{4}$ American International University-Bangladesh, Bangladesh, 17-35771-3@ student.aiub.edu \\ ${ }^{5}$ East West University, Bangladesh, naima.hassan016@gmail.com \\ ${ }^{6}$ American International University-Bangladesh, Bangladesh, ahona@ aiub.edu
}

Received Date : August 04, 2021 Accepted Date : September 13, 2021 Published Date : October 06, 2021

\begin{abstract}
The goal of the Health Monitoring System (HMS) and Textual mining is a large area to discuss. Web based health monitoring systems provide a massive number of benefits to the patient and normal healthy people to know about health issues and discuss the condition with doctors through a web portal. HMS is a process that has been used since past decades to monitor patients at hospitals in urgent situations, with the development of technologies we can use devices to check health updates of a person anytime. So, we built a prototype which is a heat sensor connected with a wireless network process. The device gives the temperature update on a real time basis, where we can add many more devices to add with the web portal and compare the data through our textual mining algorithm procedure. We believe our proposed methodology works in a larger benefit which will give a better version of the Health Monitoring System to the world for individuals. Our proposed work consists of a large amount of data that we examined to make our health monitoring system effective.
\end{abstract}

Key words: Health updates, Health Monitoring, Mental Health, Physical Health.

\section{INTRODUCTION}

In the present, modern technology has a very important role in our day-to-day life of any sector even in agriculture or mine technology or hospital service systems. Covid-19 pandemic is the most recent example of urgent medical need for the whole world. In our paper we are focusing on this problem that we have faced during the pandemic. A vast number of people needed health treatment not only for Covid-19 patients but also many other emergency patients including heart patients, diabetic patients and more, which is not protecting their health security. So, we need to build a digital online healthcare system to address this common patient condition.
Some real-time health monitoring devices have been used since past decades, where we have developed a web-based health monitoring system with the help of a real time health monitoring system. An urgent patient needs urgent solutions, so, we cover a process where a patient can communicate with a doctor or multiple doctors by using the web-based system.

\subsection{Research Background}

Health monitoring systems have been studied for decades due to the unique advantages for human health update information. Many systems have been developed for the purpose of assisting the patient in hospitals. Therefore, so many health updates giving devices have been modified for the past decades. IoT based devices, wrist watches, smart watches, oximeters and many wireless devices are providing health update information. This information helps a patient to know about the body situation. If anyone needs any treatment, the person can go to the hospital, or ask a doctor for a solution through many web-based organizations or telecommunication services. For health monitoring we might have faced the web based human health update info collection procedure, with the basic connection with the doctor.

\subsection{Problem Statement}

The world is currently going through a difficult time because of the Covid-19 pandemic. At present, in many developed countries of the world, their health system is collapsed due to the pandemic. Many doctors are not interested in seeing a patient physically because of the coronavirus, and since it can spread through airborne diseases such as sneezing, coughing etc. so that the situation has become more difficult. Currently, those who are not infected with the virus but have other diseases are also suffering. 
Because of this, they are dying in many cases due to not getting proper treatment at the right time.

We have taken this as a challenge and to solve this problem, we have designed a system through which the patient's physical condition can be constantly updated from a remote place. Not only that, our system will process the data received from the patient and will alert him about his condition, and will appoint all the doctors who are related to this issue.

\subsection{Scope of the Research}

Currently, the health system and economy of the developed countries of the world are struggling due to the Covid 19 pandemic. Many researchers are now trying to create a system that can deal with these catastrophic times. At present we must use an online system to deal with this coronavirus. If we can treat relatively fewer patients at home, the pressure on our hospitals and help systems will be greatly reduced.

We have created a system through this research by which the patient can take the advice of the doctor sitting at home. We will collect the patient's temperature, process it through the website, and provide alerts about the patient's condition as well as refer him to the concerned doctor. We will be able to inform the doctor by measuring the patient's pulse rate, blood pressure, and oxygen level by processing real-time data. This will allow the patient to be under the constant supervision of the doctor. Through this process the doctor does not have to physically come in contact with the patient, he can monitor the patient from a safe distance and prescribe accordingly. We want to increase its scope in the future. We are trying to make complete online based healthcare. We have to be careful and maintain this standard to achieve this goal and the overall outcome of this project.

\subsection{Objectives}

One of our main goals is to create a kind of health monitoring system through which patients can receive world-class treatment at home in this pandemic. We have created a system through which doctors can accurately monitor the overall condition of the patient online, even from a safe distance. We are currently working on the patient's temperature. If the patient's body temperature is normal then it will inform him and if it is higher than normal then according to our website it will give him an alert, medicine will be suggested to him and the doctor will suggest someone relevant. Later he will be able to take the doctor's appointment online. With this system, the patient does not have to go to the hospital to see a doctor and this will reduce the risk of spreading the coronavirus, meaning that those who are in good physical condition will be able to receive treatment from home. And the doctor will be able to know about his physical condition time to time. Currently, we are working only on temperature but in the future, we will also work on blood pressure, pulse rate and oxygen level through that process.

\section{LITERATURE REVIEW}

We have considered the major goal on different papers based on the Health Monitoring System and Textual Data Mining to find out some ideas of previous health monitoring systems and future areas. From the past decade according to the exigency of the medical and medicine sector there have developed many Health Monitoring systems. The improvement from older to newer is significantly noticeable. According to the study of those papers the researcher's common goal is to build a system that can easily detect patients' health information, sometimes working with the data. Some systems prefer only alarm updates. Some papers have demonstrated methods that are commonly used to detect the patient's data, alarm, update or give suggestions to the patient. Those methods are capable of detecting the patient's body information, where some common methods can measure body temperature, heart beat etc. on a scheduled time basis and also in real time. Researchers have updated some software-based systems and some device-based systems.

We are presenting reviews on some recently published papers which are specially focused on the health monitoring system and also focused on data mining processes. Selected papers are discussed briefly which include background study, background references, and some extra updates on the health monitoring system. The area of discussion was commonly accepted by all researchers but focused on newer updates, latest health monitoring updates.

\subsection{Chronological Research Background}

Health monitoring systems are dependent on devices, doctor prescription, patient knowledge, internet availability and newer updates. Some papers commonly update data from different devices but the process for health monitoring is a continuous process, so we completely focused on the present updates of health monitoring systems or patient monitoring devices. Researchers have used many devices to decide about patients' mental health update, physical health update, some specific health problem update (example-heart attack patient) Here are the studies that monitor health, patient's data, doctor's connection process, medical and medicine treatment method.

A new era of health monitoring systems ensures the healthy next generation. Developing improved health monitoring devices prevents a patient from getting affected by any disease. The concept of IOT (Internet of Things) and Cloud Computing present great advantages by providing remote and efficient services. Patients may need real-time monitoring even in rural areas, patients like heart attack patients, diabetics patients and more. A fixed health monitoring device helps those patients by informing the updates [1]. Where patients can be prevented from serious situations before reaching to some crucial stage. Devices like: 
$\varnothing$ GSM module - used for communicating computers to a GSM system.

$\varnothing$ Cloud module- Information from device transferred to the cloud so that it can be traceable to the selected devices [1].

Some of the study shows a self -monitored system of higher depression related data associated with the health monitoring app. At present time depression symptoms among young generation and different other aged people are significantly noticing day by day because of the Covid-19 pandemic, to gather the information of metal depression-related problem the asking health information usage was low whereas focusing the question from persons mental health related question that can define depression situation. By using 12 depression related topics it was so promising because the smartphone app was placed to update the information as researchers have mentioned its main purpose [2].

Occupation related health problems are common for the patients who are working in underground mines. In the past it was very difficult to volunteer those workers to give proper treatment and also to take an update of their health. It is now possible to maintain track of mine individual and their health characteristics with the technological improvements. Volunteers are now highly dependent on new advanced technology. Wearable health sensing systems are an alternative solution to these problems, and they can offer information on miners' health status [3].

A health monitoring system based on several individuals' actions. Some social needs are also important to make a supporting free clinical basis health monitoring system. To make sure that the intern doctors give the patients a solution sometimes. Among 223 patients screened, 66.4\% $(\mathrm{n}=146)$ most of them accepted the need of prescription from interns. Different age's patients have different health related problems according to age, gender, race, education, and primary knowledge about health [4].

\subsection{Methodological Research Background}

In this paper [5] we have found some of the very updated methods discussed with a couple of previously used method combinations and specific explanations. Hand motion is an important component, which plays a key role in hand function assessment in different areas for health treatment. As discussed in the literature, it is difficult to capture accurate hand motion, particularly in combination with sensor topologies placed on a textile or an exoskeleton device. Smart gloves require time-consuming and monotonous calibration to increase the device's overall accuracy during operation. Consequently, anthropometric dimensions have significant differences among individuals, including hand size, fingers, length and thickness. Glove-based systems must then be associated with various finger joint locations for various people, which would have an influence on the overall measurements accuracy [5].

Mental health is a very effective part of the health monitoring system. A mood tracking method is a solving process for mental health solutions. Nowadays smartphone apps are capable enough to detect a person's behavior, situations, likes, dislikes and more. An app used in this method Evidence on user engagement with self-monitoring apps and their effects on health is still limited (Rubanovich, Mohr, \& Schuller, 2017) and virtually absent in conjunction with health information seeking. However, Montagne, Cariou, Feuillet, Langlois and Tzourio (2018) report that in a French sample, only a few individuals $(72 / 498, \sim 14.5 \%)$ used at least one health-monitoring app to look up health information, while virtually everybody (450/476, 94.5\%) had searched online for health-related information in the previous year, including information about depression (243/476, 51.1\%) [2].

Based on the method of college students' physical health monitoring system this system allows the student to know about their precautionary results, also combined with previous methods in a simpler way of calculative formulas. Taking some comprehensive data from college student physical health as input and continuing the input to convert an easier educational result [4].

Extreme situations or places need a special kind of health monitoring system. The previously normal mining system, industrial sectors, deep ocean workers health monitoring was difficult to measure because of the environmental issues. Several different research fields deal with text, such as text mining, computational linguistics, machine learning, information retrieval, semantic web and crowdsourcing. The research emphasizes that many people (over 800,000) were forced to take time off work for an average of eight working days per year [3]. Three TelosB sensor motes operating at 2.4 $\mathrm{GHz}$ signal frequency were used to accomplish the experimental measurements in mine galleries. A laptop was used to save the collected data and was also used for post-processing [3]. Wireless signal characteristics, underground wireless connectivity channels connecting methods discussion. The effect of tunnel geometry, as well as additional losses owing to miners' mobility and noise caused by mining machines, have been discussed by researchers [3].

Smart health monitoring evaluation started many years ago. During treatment in the hospital, a patient with a heart infarct (65 years) received measurements of body and excretion weight using the sensor system set up in the rooms. Researchers examined at how the treatment affected the recovery of circadian rhythm for weight change. Two SAS patients (45 and 56 years old) had their respiration measured while sleeping with a flat-type sensor under the pillow, and apnea and hypopnea were discovered by the decreasing of the respiration signal. Researchers also evaluated its detection accuracy by recording polysomnography (PSG) at the same time [7]. 


\subsection{Existing System Background}

At present time call center based, special device based and other special occupational organization-based health monitoring is very active. The hospital health monitoring is updating day by day but we are focusing on the individual health monitoring system.

In many countries several call centers are working on health services through call centers. They connect the patient with an intern doctor or a specialized doctor to give a primary suggestion for the patient. Even some services include a patient and call center communicator connection on a regular basis. If a patient is in surgery or any other ongoing treatment the hospital provides the call center for the patient to inform about the medics and more updates if needed.

The call center was subsequently set up with a local private telecom partnership. At the early days of call center service, the objectives for the design and implementation of the call center intervention were to improve maternal and neonatal health outcomes in the Greater Accra region by: (1) Facilitating the referral processes for maternal and newborn emergencies primarily (2) Providing reliable expert advice to frontline healthcare providers, (3) Identifying and assuring sustainable and affordable local financing for the recurrent maintenance costs of the intervention [8] [23].

In terms of time and storage, several existing systems for identifying user behavior are extremely sophisticated. This technique focuses solely on manual assessment, which takes time and does not yield promising results because the user's emotions fluctuate depending on personal and situational issues. The current system does not employ biometrics to recognize the users' emotions. With psychometric test-based automatic individual criminal behavior prediction, the current system does not focus on user facial expression. [9].

Device based health monitoring is widely used nowadays. At present so many smart watch companies provide heart rate count systems, blood pressure, blood temperature, sleeping time schedule, body fat count, steps count system and many more which are included in the health monitoring system (HMS) [10].

Occupational update-based systems have different kinds of health monitoring devices. Some special IoT devices, hand bands or specific kinds of company owned devices may help the work of health monitoring. Example wise we may see underground mining workers have different types of devices rather than deep ocean workers.

\subsection{Theoretical Framework Review}

A strong network used for social networking to enhance the communication among different IoT items. SIoT which stands for Social Internet of Things making a new paradigm, The new framework for the integration of e-health and SIot has been proposed for all generic symptoms such as most common allergies and sickness through some widely-data updates. Now a days smartphones are manufactured with a large number of IoT-enable sensors which are discussed below-

\subsubsection{IoT Based Framework}

Health Monitoring and Medical information system based on IoT integral technologies such as mobile computing and wireless networks. Its aim is to provide a patient with remotely receivable sensing, sound, image, video multimedia information, which will enhance the medical diagnosis accuracy and the clinical service quality. By wearing necessary equipment, a patient can update formation accurately of -blood pressure, heart rate, body temperature, pulse, and other information. This information can be transmitted through some technologies such as -Zigbee, Wi-Fi, Bluetooth etc. [11].

\subsubsection{App Based Framework}

App based questions asked to the person to detect the emotional and mental condition with some pre-setup questions.

\subsubsection{AI Based Framework}

Facial expressions are not only the most natural way of expressing human emotions, but they are also an important non-verbal means of communication. Effective methods for automatically recognizing these facial expressions can be developed, and significant advancements in the field of human-computer interaction can be made. In the hopes of achieving these improvements, facial emotion recognition research has been conducted. In reality, automatic facial expression recognition can be used for a variety of additional purposes. Artificial Intelligence has long depended on facial expression recognition to get insight into how to accurately model human emotions in robots. Recent advancements in this field have prompted researchers to broaden the scope of facial expression identification to include chat room avatars and video conferencing avatars [12].

\subsubsection{Automated Framework}

Fully automated health-care monitoring system without attachment of any biological sensors set up at the hospital room. The sensors are placed in the toilet, bed, and bathtub, and the data collected is automatically evaluated and shown using monitor equipment that amplifies the sensors, computer, memory, LCD, and LAN module. The server equipment stores and displays the analytical results of each sensor, such as a trend-chart [13]. 


\section{PROPOSED METHODOLOGY}

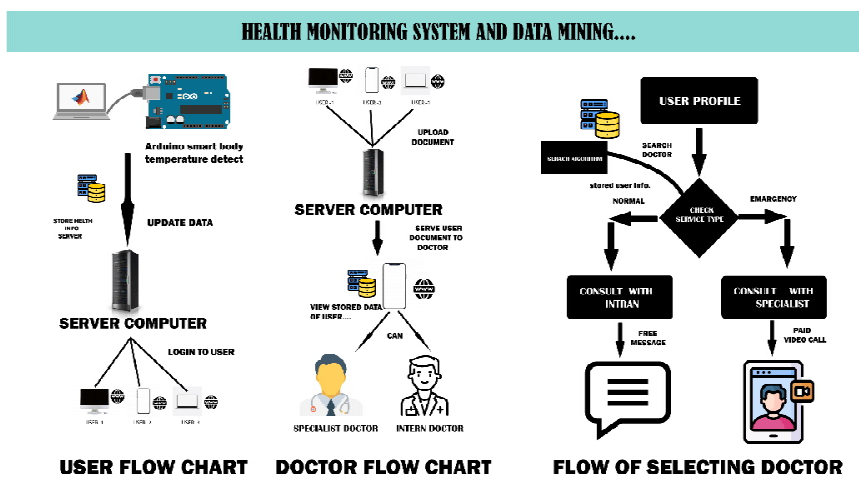

Figure 1: Health monitoring system

First, we have tried to work with different smartwatches, but we have faced some problems getting data from the device because of security issues. Almost All the reputed companies are very concerned about their security system; they don't allow others to access their user data. As we cannot use their data, we have developed a new device which can communicate with our web application. Our device can measure user body temperature and send it to the web application. This device can send data in actual time. We can measure data quickly and accurately. The device Accuracy Rate is approximately $98 \%$ with the other devices. When the user data is sent to the server, then it will analyze the data and give a notification to its users, whether it is in a safe zone. If The user's health condition is bad, then our application will detract it and suggest some medicine and best doctor depending on the user's health issue.

\subsection{Working Procedure}

In that flow of work, we have represented the working procedure of the device and its users. At first, the Arduino device will detract from the user's body temperature using its sensor. It can send data to the server in the user's table. The server will store it and display it to its user. Users can access it using browsers from the server anytime and anywhere. Users can upload their documents and death status manually. We have developed a device for body temperature but if any user uploads their pulse rate, blood pressure, and sugar level our application can analyze the data of the user. After analyzing the user data, it will suggest some medicine and the best doctor depends on the user's problem. Users can make appointments with the suggested doctors.

Doctors can see all the appointment requests of current dates. The doctor can approve appointment requests. As it is online, Doctors can consult with the patient any time using a chat or video call. Doctors can check the user's health status and upload documents.

Here are two types of doctors: specialist and intern. Users can choose one of them if anyone wants to consult with an intern. It's free of charge. Free facilities will in general serve patients with social requirements, few are screened or get help. Absence of faculty, assets, and strategies to recognize and help patients are reasons not many free facilities think about friendly requirements. To address this assistance hole, a midwestern free facility and adjoining Social Work program set up an organization. A social necessity screen was formed and coordinated into medical care practice [14]. For a consultation with a specialist, users must pay. After payment, the user will get a notification of the appointment date and time. Users have to make themselves available. The doctor will call the patient.

\subsection{Comparison}

In this section we will compare the data process with the other documents.

Table 1: Comparison with another web application

\begin{tabular}{|c|c|c|c|}
\hline Title & $\begin{array}{c}\text { Health } \\
\text { Monitoring } \\
\text { System }\end{array}$ & Health Plus & $\begin{array}{c}\text { Digital } \\
\text { Hospital }\end{array}$ \\
\hline $\begin{array}{c}\text { Project } \\
\text { Address }\end{array}$ & Our project & $\begin{array}{c}\text { http://healthplu } \\
\text { s } \\
\text {.life/\#/hom }\end{array}$ & https://care.dh \\
health/
\end{tabular}




\subsection{Web-Application Discussion}

The separate discussions of our HMS with the working example of our self-built web-application are given below:

\subsubsection{Home Page}

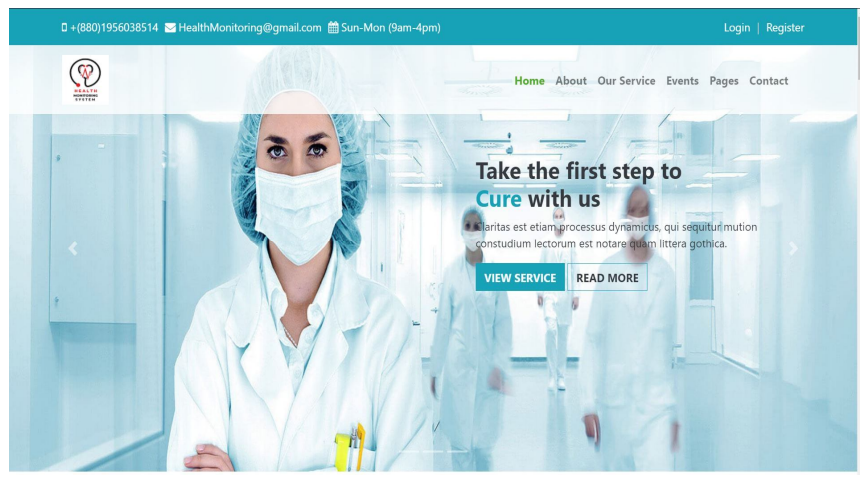

Figure 2: Home Page

A home page is the first impression for any user. Our Home page is very user-friendly. On this page at glance, any user can understand what kind of application it is. Users can get ideas from our FAQ pages that will help to get solutions to his/her problem. On the home page, users could see all kinds of services. They could get lots of health tips on the home page. Users can see all kinds of updates on the home page (Figure: 2).

\subsubsection{User Type}

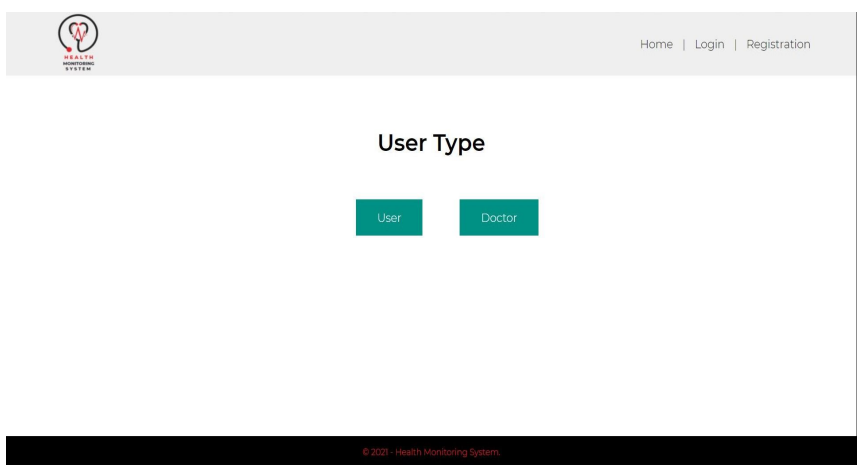

Figure 3: User Type

In our entire project, we are working with three users. From these only two users can register by themselves. Patients and doctors can register manually but the admin has the right to check user information. Using this page, both doctor and patient can choose their user type easily. There are two buttons for two types of users (Figure:3).

\subsubsection{User Registration}

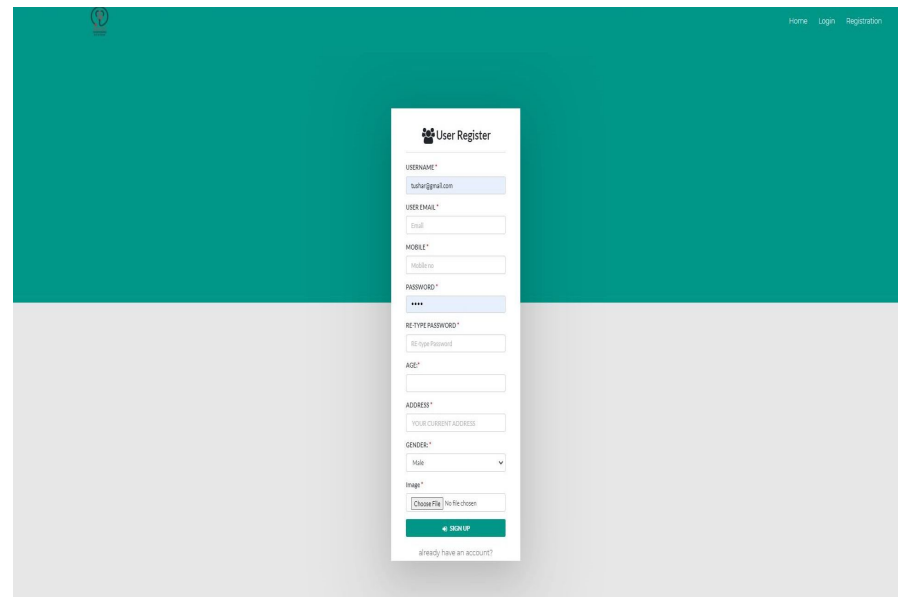

Figure 4: User Registration Page

This registration page allows users to register themselves easily. It is very interactive and user-friendly which will help its user to insert their information with a correct description.

Utilizing our proposed wellbeing observing structure, a brilliant property holder or specialist co-op can make a digital actual space with a protected advanced wallet for every human occupant and approved IoT wellbeing gadgets [15].

Users should have to insert their data correctly because if they put wrong information, they will get the wrong suggestion. Every information of a user is very important because our system will analyze (description of the analysis is defined at the view data part) users given data.

\subsubsection{Doctor Registration}

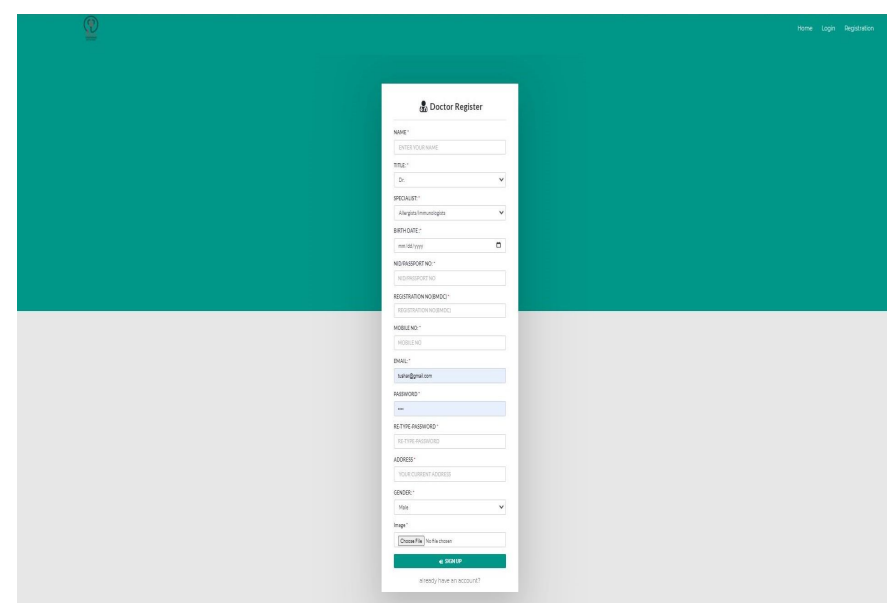

Figure 5: Doctor Registration Page

On this registration page, any doctor can register on their own. It is possible to register a real doctor because we are taking some vital information from the doctor (Using BMDC). With this information, it is possible to check whether this doctor is fake or not. Doctors can select their special scope of work. 


\subsubsection{User Login}

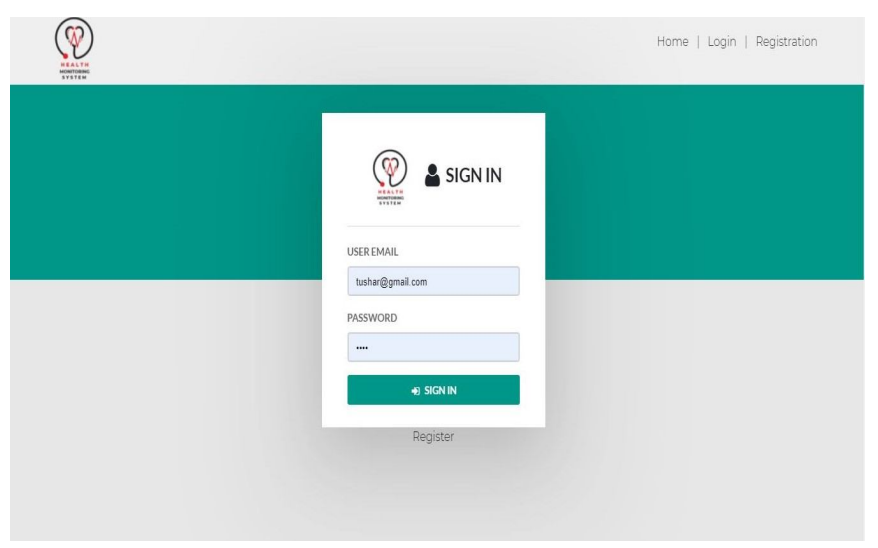

Figure 6: Sign In Page

This is a multi-user login page. Using this login page all the users' can log in to their profile entering their registered email and password. If any input is wrong, the user will get an alert on the page (ex: invalid email or password). If user input is correct, then they will get access to enter their profile.

\subsubsection{User Dashboard}

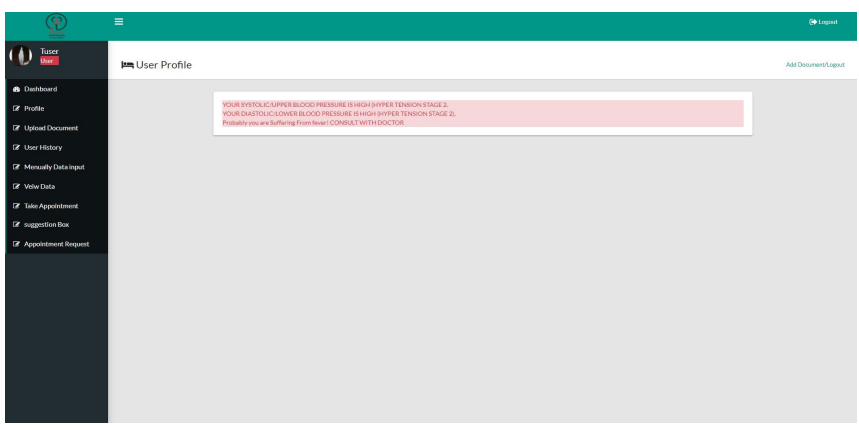

Figure 7: User Dashboard Page

After a successful Login user will see his dashboard. User health status is always updated in the dashboard. This must help to be aware of health.

\subsubsection{Report Input File}

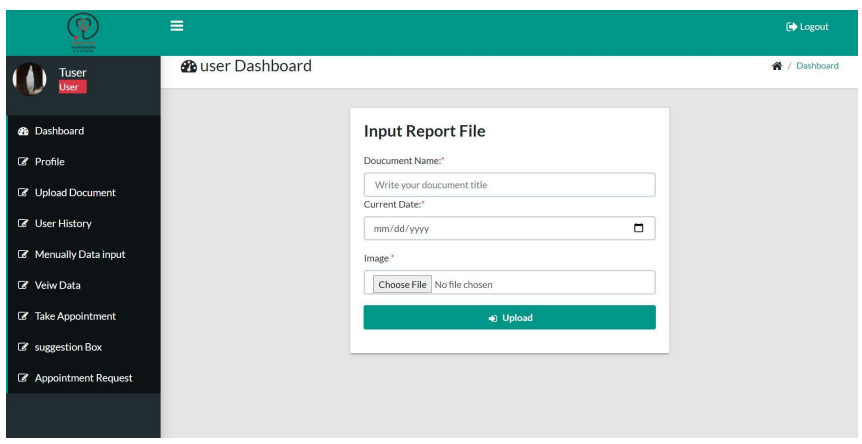

Figure 8: User Document Upload Page
For monitoring any user health status lots of information is needed. Using this page, users can upload all kinds of important documents. If a user uploads their document here, they can easily access all the information from any location through his ID and password. The patient does not have to carry all the medical test reports with him which will greatly reduce the patient's suffering.

\subsubsection{Take Appointment}

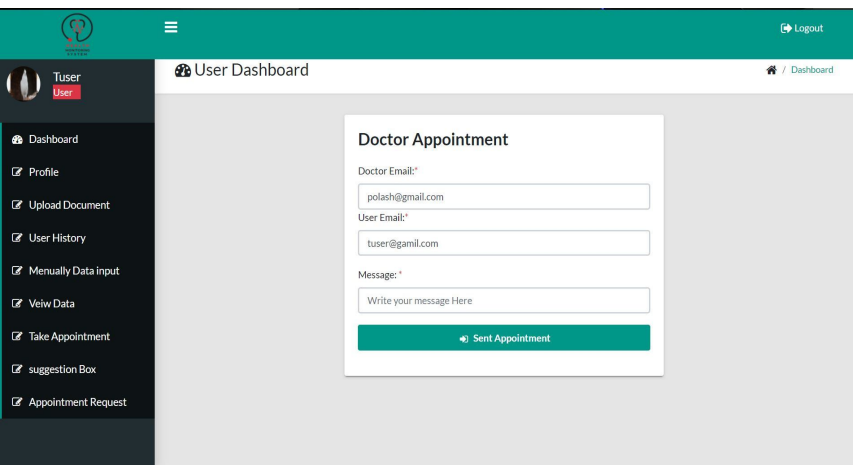

Figure 9: Doctor's appointment Page in User Dashboard

Using this feature, users can send appointment requests to doctors. This is a free procedure. In this pandemic situation, that could be very helpful to all.

The point is to configure, carry out and assess a versatile application that can help seniors in dealing with their everyday home lives. Attempting to persuade them to take their prescription, evaluating their pulses and educating them to move and screen their developments. Also, help specialists track their patient's wellbeing information by sending them day by day persistent pulse information [16].

If anyone sends an appointment request using this, he/she can send a short description message to the doctor by the message option, the doctor will get an idea of the user's problem.

\subsubsection{Sent Data to Server}

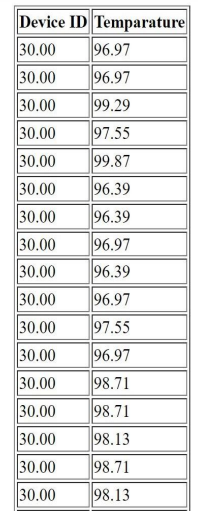

Figure 10: Device Value

Here is some user data that our device has detected. All the devices have a unique id. Our device will send data in the database using a unique id. 
This device will send data to the database if the body temperature is over 96.5 and repeat the same data over two times.

\subsubsection{User Profile}

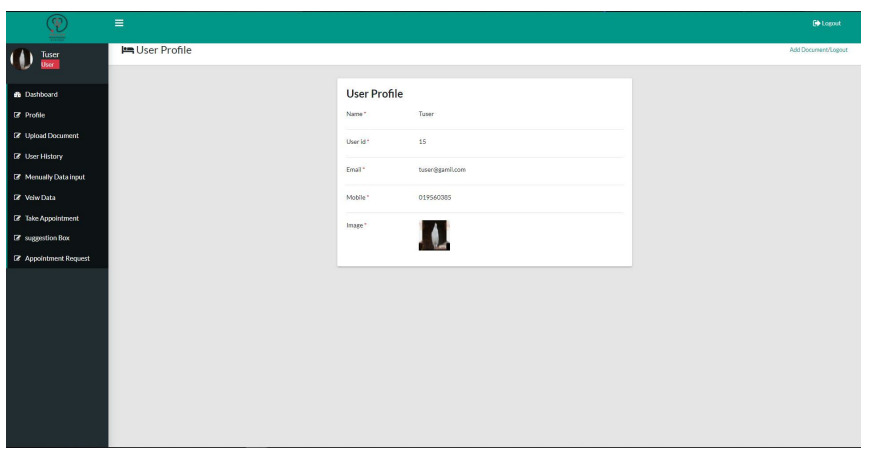

Figure 11: User profile Page

In the user profile page, all the information will be shown. Users can delete his/her profile or edit their information using the edit option.

\subsubsection{View User Health Status}

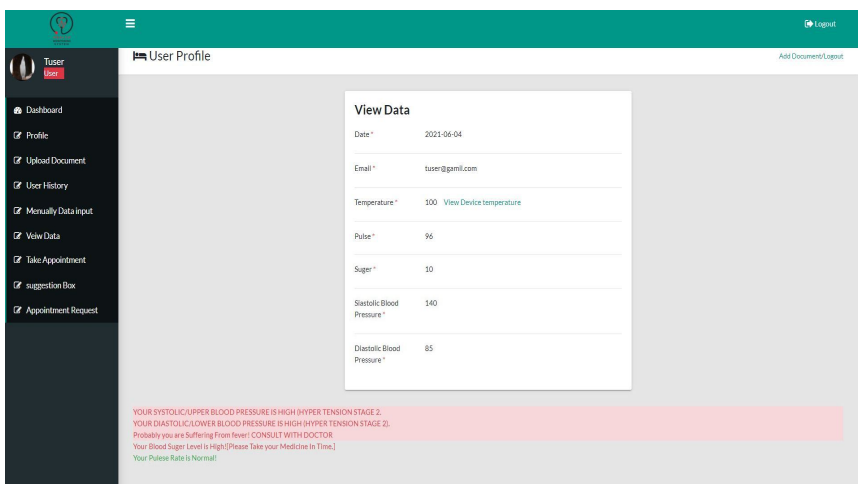

Figure 12: User profile (View Data) Page

On this page, users can see their current health status depending on their current data. We completed our algorithm for body temperature, pulse rate, sugar level, and blood pressure. Our application always works on the last input data. All the red sentences are an alert for users because these results are above or below normal. Green Sentence Means the result is normal.

The plan of the system gives a viable patient wellbeing checking method which uses temperature just as heartbeat rate locators to screen patient's wellbeing and uses web office to insinuate specialists of the matter assuming any. The indicators get interfaced to a microcontroller to check the patient's condition which is associated with LCD show and Wi-Fi framework to illuminate messages [17].

As the shrewd cultivating applications are acquiring research ground to convey nonstop perceivability into soil and yield wellbeing plan of Intelligent IOT based brilliant coconut tree wellbeing observing gadgets are proposed since utilization of astute tree wellbeing checking frameworks (gadgets) makes the undertaking of coconut cultivating still simpler [18].

\subsubsection{Check Appointment Request Status}

Appoint Request Status.

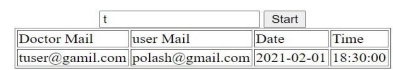

Figure 13: Appointment Request Status

On this page, users can check their appointment request status (approved or not). This is very easy to check because we have used ajax to find user requests. Ajax is a very efficient, fast and modern system for searching data from database.

\subsubsection{Doctor Dashboard}

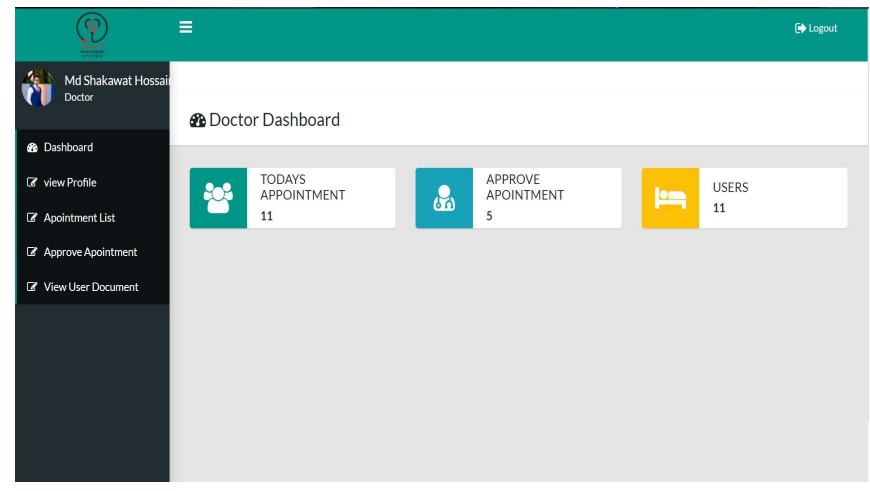

Figure 14: Doctor Dashboard Page

Every doctor has their dashboard. On this page, doctors can see everyday appointment requests, total approved appointment requests and the total number of users who have consulted with him/her. All the doctors will get an initial brief using this page.

\subsubsection{Doctor Profile}

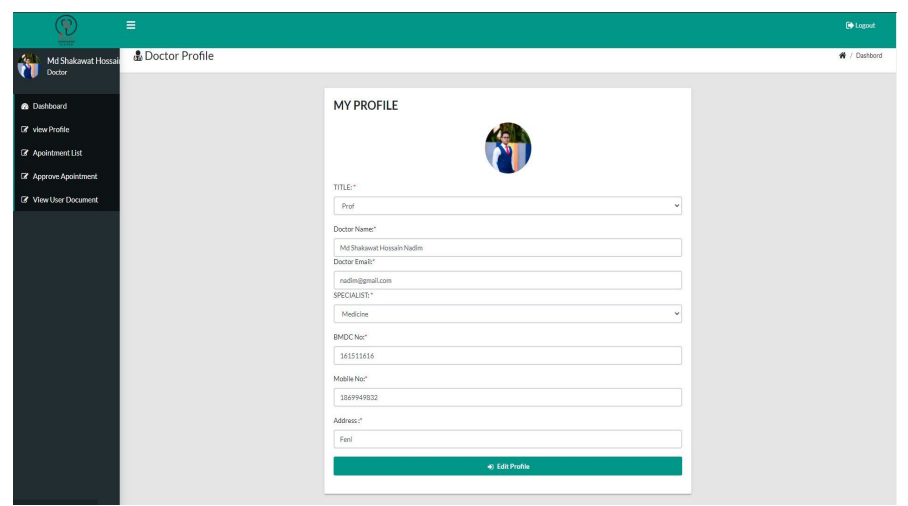

Figure 15: Doctor's Profile Page 
We have completed lots of our operations based on doctor's information. Doctors can update their information like title, email and cell phone number etc. using the edit option of the profile.

\subsubsection{Approve Appointment}

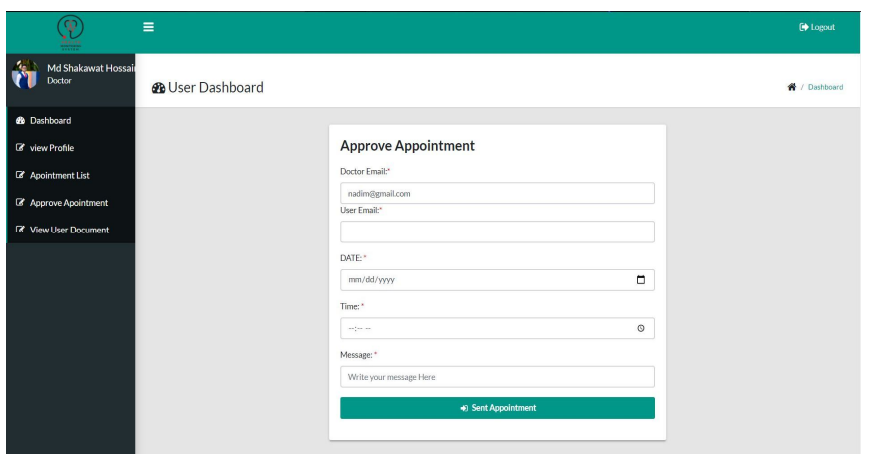

Figure 16: Approval of Appointment Page

Doctors can approve all the appointment requests using this page from the request list. Here are five text boxes for approving appointment requests. It will automatically fill user email and doctor email addresses from the appointment list. Doctors can send a brief message to users.

\subsubsection{Search Document}

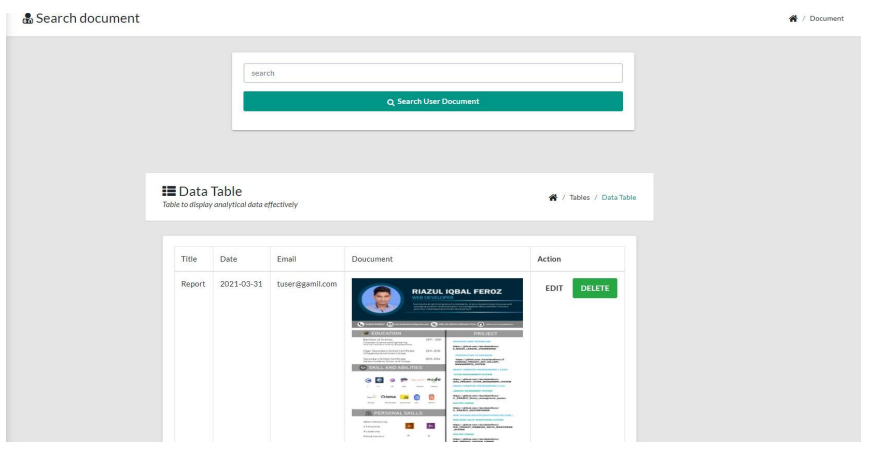

Figure 17: Search Document Page

This is another feature for doctors with this feature, doctors can see all the uploaded documents of the specific patient. Documents can be png, jpg, pdf etc. This will make the treatment system easier.

\subsubsection{Appointment List}

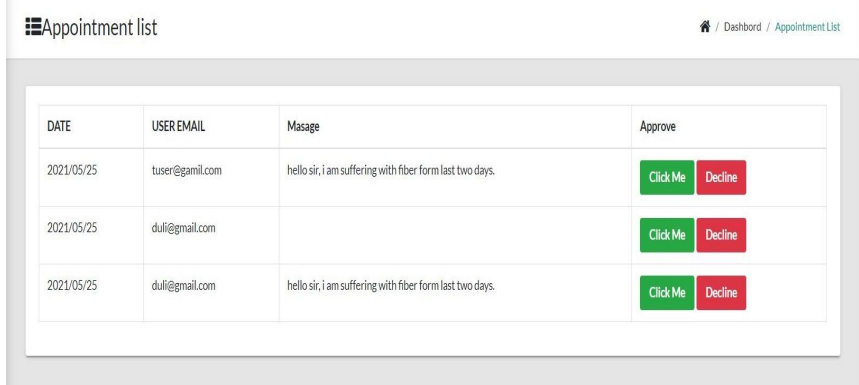

Figure 18: Appointment List Page
On this page the doctor can see all the appointment requests. Doctors can approve or decline the requests. If the doctor wants to decline the request just need to click on the decline page otherwise click on the approve button. After clicking on the Approve button, the Approve appointment page will be open.

\subsubsection{Admin Dashboard}

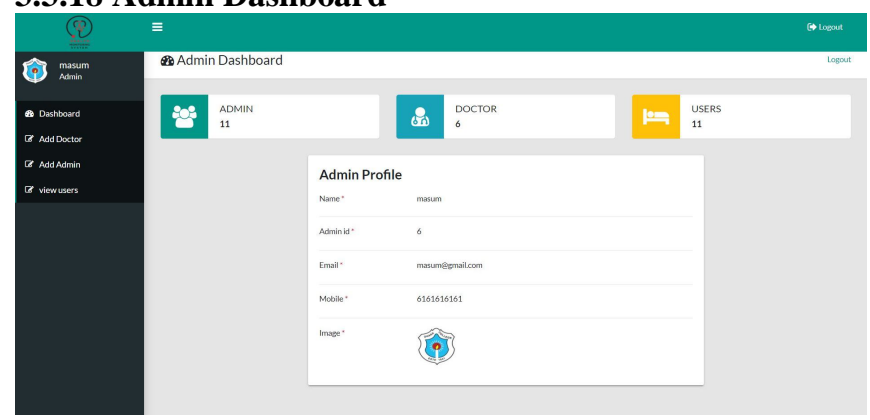

Figure 19: Admin Dashboard Page

Admin is very important for any project/company. Admin has authorization to see some crucial information and edit these which others do not have. All the admin can change user info based on user requests. In this page admin can see his information and number of users of the application (ex: number of doctors, admin and users). Admin also can see their registered information here.

\subsubsection{Admin Registration}

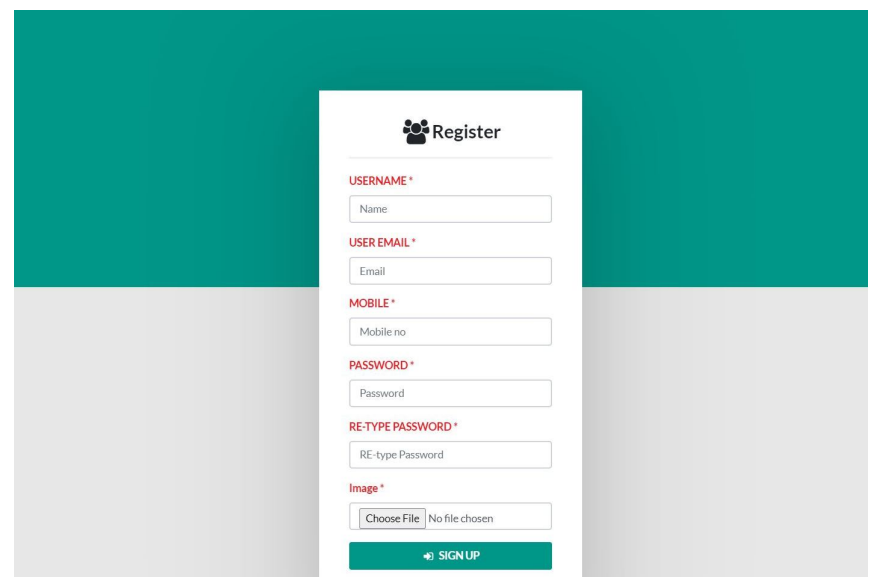

Figure 20: Admin Register Page

Only the admin can register a new admin. This admin post is very vital and highly secured because the admin has some special power. By using this post anyone can hamper project owners. That is why only the admin can access this page. To register a new admin this information is needed. Using this information admin can $\log$ in. 


\subsubsection{Admin Activity}

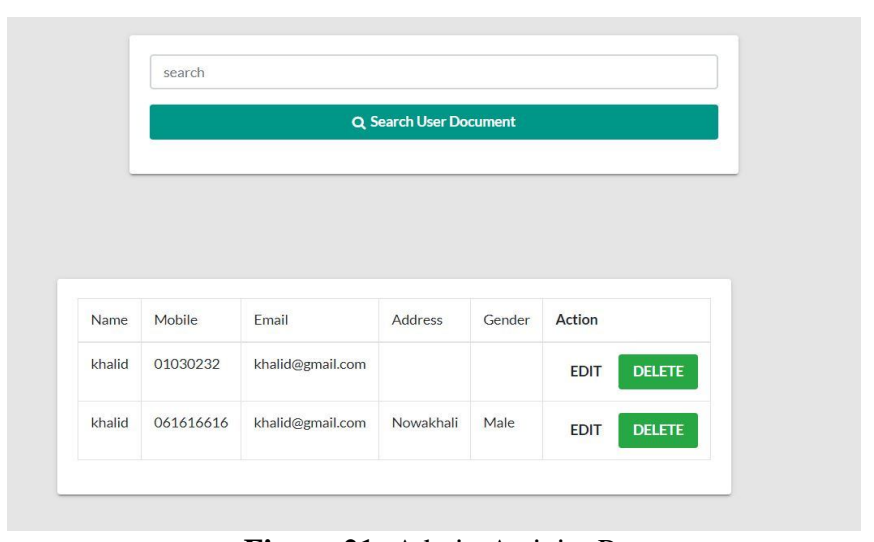

Figure 21: Admin Activity Page

This is the activity page for the admin. If anyone complains then admins have that authorization to ban or suspend users' profiles. Admin should inform users first if he/she doesn't agree with the terms and conditions then they will take action against him/her. Admin can find any user's information; it can be admin, doctor, or patient.

\section{RESULT AND DISCUSSION}

From this portion we will test the functionality and effectiveness of our system. We have worked with the patient's body temperature, blood pressure, blood Sugar, and pulse rate in our system. The body temperature is determined by the device and that temperature is analyzed using the algorithm of our system. We can also analyze data on blood pressure, blood Sugar and pulse rate. Then we provide real-time results and suggestions based on their analyzed data. We have analyzed the different types of data received from the device to increase the efficiency of our system. For performing this project, the code was written in PHP using the visual code sublime text. Also we are using some php built-in functions like- ReflectionType(), ncurse_termname(), get_defined_functions() and we are using Sass and bootstraps for high quality design.

\subsection{Database}

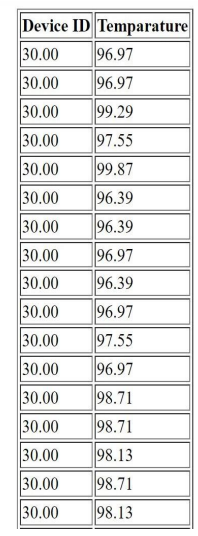

Figure 22: Device Data

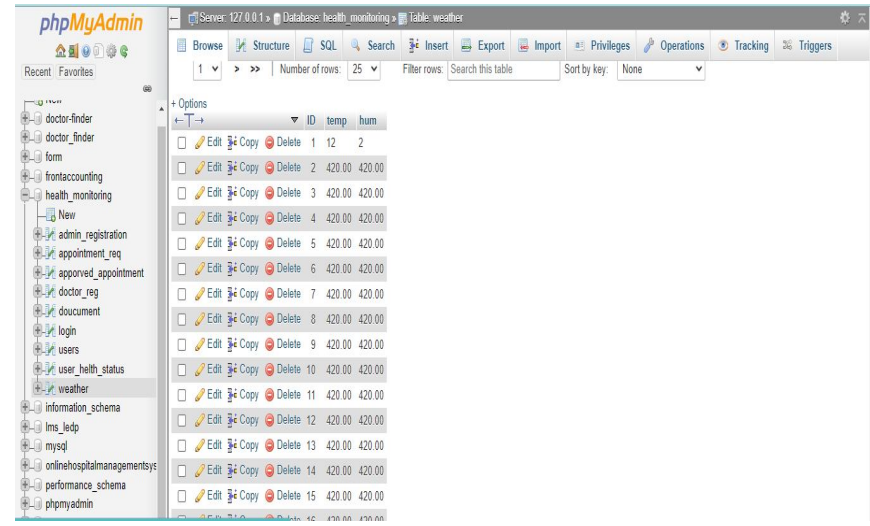

Figure 23: Database

All data from the device will be stored in our database. First, we create a database table specifically for all users. There are two ways to input data into the database. Firstly, we can take data input from the device and secondly, we can take input manually. The data in the database will be filtered and divided into two parts. The first part will have the data of the device and the second will have the data that was taken manually. Then we will fetch the data from the database and analyze it in our system with the algorithm that our system has. Algorithm set separately for device input data and manual input data. We have used linear search algorithms and Hash tables to do the data analysis. And here we are using two types of databases, a localhost and a health monitoring live-server. Manual data we input to the localhost server and we upload device data to the health monitoring live-server.

\subsection{Experimental Result}

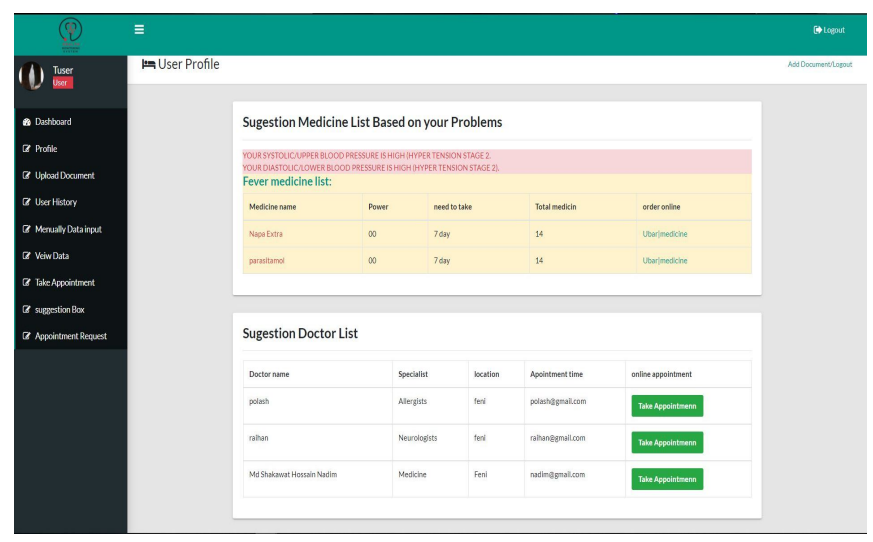

Figure 23: Suggestion of Medicines and Doctors

Our approach is mainly web-based. All the data is getting validated and updated on the server. The application has a diagnostic capability however due to the limitation of time we are doing it mainly on the temperature only using the device. The data is collected using the thermal sensor. After a couple of readings, we have come to realize that, taking reading using Node MCU will result in wrong data which is outside the actual plausible range of data. So, we are taking 50 sets of 
data per patient which ensures that we can remove the outliers since those will be beyond the actual data result, which means we are achieving an accurate result in this experiment. On the other hand, the data is mined to see a pattern in the fluctuation in fever to determine whether the fever is from influenza or some serious problem. We were successful at doing so by comparing the data with the previously accurate information of the previous patient and figuring out in which range of normalization does the actual fever fluctuate and predict the result. After mitigating these issues, the experimental result shows a successful run of the code. However, more comprehensive experimental study is required to get a more robust result.

With this system, a user can easily monitor his body. As a result, it can be understood what disease has really occurred. And if so, the user can see suggestions on what they should do. The most important thing is, there is no need to go to the doctor for any minor ailment and if their disease level is serious, our system arranges a specialist doctor's appointment directly. Using this application, users can take emergency suggestions from specialists. In an emergency case, a small suggestion can save any patient's life. Our data analysis system should help any patient to decide faster.

\section{CONCLUSION AND FUTURE WORK}

\subsection{Conclusion}

We have worked on how to transform the health system completely online through this web-based health monitoring system. As a result, the physical condition of a patient can be easily observed from a distance. This means that if the physical condition of all the illnesses is not very critical, he will be able to take treatment at home and this will reduce the chances of transmission from one patient to another. With this system, the doctor will be able to make decisions accordingly by observing the patients through real-time data. This means that when the patient visits our system, it will be connected to our database via internet Wi-Fi or cable. And this data will be stored directly in the database. Our system will analyze the data and send the message according to the patient's condition. In other words, when his condition is serious, he will show it and refer the patient to the departmental doctor according to the type of his illness. The doctor will be able to access all the data of the disease when a patient needs a doctor's appointment.

On the other hand, the pressure on our hospital and health system will be greatly reduced. This means that through our system, a patient will be assured of high-quality health care at home. During the current coronavirus epidemic, when a patient goes to the hospital for treatment, another healthy person is more likely to be infected. So, if we can ensure this health monitoring system at home, the chances of spreading any diseases will be reduced.

\subsection{Future Work}

The features of our project have already been discussed. We are currently only working on Human Body Temperature and doing real-time analysis and suggesting the doctor in this sector. But in the future, we will be able to work with real-time data through the patient's pulse rate, blood pressure, amount of oxygen in the body and so on.

\subsubsection{Connect Multiple Devices}

We have a desire to connect more and more new devices through which patient's sufferings will be greatly reduced. We will be connecting here a variety of devices to detect blood pressure, pulse rate, and oxygen level in addition to temperature sensors. Each device will have a specific number that will be integrated with the patient's ID, meaning that a patient can connect multiple devices to his ID. And each device information will be linked separately to our website under the same patient database. In the future, data will be collected through these devices, analyzed through our website, and referred to the right doctor.

With assembly of computerized innovation and keen gadgets, checking and following information continuously become valid. Given the innovation of numerous well-being crucial sensors and action GPS beacons, powerfully measuring and checking of fundamental wellbeing boundaries become simple. The sensors and gadgets utilize various conventions and specialized techniques for communicating the information [19].

\subsubsection{Taking an appointment with a foreign doctor}

Through this system, he will be able to receive world-class services in the country. In other words, he will be able to sit in one country and get a checkup by appointment from any doctor around the world. In the future, we will increase the number of doctors based on patient feedback. We will be able to access them through an application and analyze the results of that application.

Advancement and assessment of intensive care unit (ICU) choice emotionally supportive networks would be incredibly worked with by the accessibility of a huge scope ICU patient information base. An incorporated easy-to-use social data set was created for perusing patients' clinical data lab results, liquid equilibrium, prescriptions, medical caretakers' advancement notes. They will prove to be important information for patient monitoring research and will support efforts in medical data mining and knowledge discovery [20].

\subsubsection{Developed Mobile Application}

Currently, most people use smartphones, so we will create mobile application software apps. There will be a notification alert system that will automatically issue alerts through this software when physical condition deteriorates. A mobile 
application was created for diabetic patients through which they could monitor the physical condition of the patients. This application could add to the redesign of diabetes the executives and the vast majority of them said that they would positively utilize a diabetes application to oversee patients whenever it is carried out. Besides, doctors could invest a piece of their energy, imparting data to their diabetic patients through this App [21].

Through Our apps, the patient or the relatives of the patient can monitor his physical condition regularly. This data must be added to our database server so that the doctor will be able to access the data when the patient makes an appointment with the doctor. Since we will have all the information of the patient, we need to ensure the security of the database and mobile application on our web server. Adding all the features to this mobile application will make it a modern facility as a complete health monitoring system.

\subsubsection{Balanced Diet}

Since many patients will have personal information stored here, a chart of balanced diet that the patient needs to recover will be prepared based on his or her physical condition. They will be given an idea of what kind of hygiene they should follow after recovering from the disease.

The shortfall of food checking has contributed essentially to the increment in the populace's weight. Because of the absence of time and occupied schedules, a great many people don't control and record what is burned-through in their eating regimen. A few arrangements have been proposed in PC vision to perceive food pictures; however, few are worked in healthful checking. This work presents the improvement of an astute framework that characterizes and portions food introduced in pictures to help the programmed observing of client diet and dietary admission [22] [24].

When a patient recovers and if there is a possibility of disease, later on, he will be able to know from the beginning. Many incurable diseases in the world can be cured if we detect them early. By connecting such devices to our systems in the future, we will be able to give patients an early idea of future disease transmission through the constant monitoring of patients. Different types of symptoms appear before a major illness occurs and if we can think about them properly, they are more likely to recover. Our system will analyze his physical condition and help him to make decisions accordingly.

\section{REFERENCES}

1. C. Premalatha, R.P. Keerthana, R. Abarna , "HUMAN HEALTH MONITORING SYSTEM" International Research Journal of Engineering and Technology ,Volume: 06 p-914-916,Jan 2019, p-ISSN: 2395-0072.

2. Sebastian Scherr and Mark Goering, Is a self-monitoring app for depression a good place for additional mental health information? ecological momentary assessment of mental help information seeking among smartphone users, Health Communication 35 (2020), no. 8, 1004- 1012, PMID: 31025888.

3. A. Ranjan, Y. Zhao, H. B. Sahu and P. Misra, "Opportunities and Challenges in Health Sensing for Extreme Industrial Environment: Perspectives From Underground Mines," in IEEE Access, vol. 7, pp. 139181-139195, 2019, doi: 10.1109/ACCESS.2019.2941436.

4. Jeannine M Rowe, Eunyoung Jang, Victoria M Rizzo, Yeongmin Kim, Bonnie Ewald, Matthew R Vail, and Katherine Drechsler, Supporting free clinic patients' social needs with MSW interns: A pilot study, Social Work in Health Care 0 (2021), no. 0, 1-20, PMID: 33657981 .

5. Henderson J, Condell J, Connolly J, Kelly D, Curran K. Review of Wearable Sensor-Based Health Monitoring Glove Devices for Rheumatoid Arthritis. Sensors. 2021; 21(5): 1576.

6. Y. Jiao, "Design of College Students' Physical Health Monitoring System Based on Big Data Platform," 2020 12th International Conference on Measuring Technology and Mechatronics Automation (ICMTMA), 2020, pp. 733-738, doi: 10.1109/ICMTMA50254.2020.00160.

7. L. L. Chan, B. G. Celler and N. H. Lovell, "Development of a Smart Health Monitoring and Evaluation System," TENCON 2006 - 2006 IEEE Region 10.

8. Oduro-Mensah, Ebenezer, et al. "Implementation of a referral and expert advice call Center for Maternal and Newborn Care in the resource constrained health system context of the Greater Accra region of Ghana." BMC Pregnancy and Childbirth 21.1 (2021): 1-16.

9. Veena, S., and John Aravindhar. "Mental Health Monitoring System Using Facial Recognition, PEN Test and IQ Test." (2021).

10. K. K. F. Tsoi et al., "Blood Pressure Monitoring on the Cloud System in Elderly Community Centres: A Data Capturing Platform for Application Research in Public Health," 2016 7th International Conference on Cloud Computing and Big Data (CCBD), 2016, pp. 312-315, doi: 10.1109/CCBD.2016.068.

11. Ru, Lei, et al. "A Detailed Research on Human Health Monitoring System Based on Internet of Things." Wireless Communications and Mobile Computing 2021 (2021).

12. Aiswaryadevi, V. J., et al. "Artificial Intelligence and IoT Framework for the Health Monitoring System." Artificial Intelligence and IoT: Smart Convergence for Eco-friendly Topography 85 (2021): 35.

13. K. Motoi et al., "A fully automated health-care monitoring at home without attachment of any biological sensors and its clinical evaluation," 2009 Annual International Conference of the IEEE Engineering in Medicine and Biology Society, 2009, pp. 4323-4326, doi: 10.1109/IEMBS.2009.5333712. 
14. J. K. Reena and R. Parameswari, "A Smart Health Care Monitor System in IoT Based Human Activities of Daily Living: A Review," 2019 International Conference on Machine Learning, Big Data, Cloud and Parallel Computing (COMITCon), 2019, pp. 446-448, doi: 10.1109/COMITCon.2019.8862439.

15. Rahman, Md Abdur, et al. "A Natural User Interface and Blockchain-Based In-Home Smart Health Monitoring System." 2020 IEEE International Conference on Informatics, IoT, and Enabling Technologies (ICIoT). IEEE, 2020.

16. Al-Shaher, Mohamed Adel, and Ali Qasim Abdul-wahed. "Design and Implementation of Android Application to Thi-Qar Doctors Guide and Resident Pharmacies." 2020 4th International Conference on Electronics, Communication and Aerospace Technology (ICECA). IEEE, 2020.

17. Hartalkar, Aditya, et al. "Design and Development of Real Time Patient Health Monitoring System using Internet of Things." 2020 IEEE 1st International Conference for Convergence in Engineering (ICCE). IEEE, 2020.

18. Sridhar, B., S. Sridhar, and V. Nanchariah. "Design of Novel Wireless Sensor Network Enabled IoT based Smart Health Monitoring System for Thicket of Trees." 2020 Fourth International Conference on Computing Methodologies and Communication (ICCMC). IEEE, 2020.

19. Kesavan, Selvaraj, and Ganesh Kumar Kalambettu. "IOT enabled comprehensive, plug and play gateway framework for smart health." 2018 Second International Conference on Advances in Electronics, Computers and Communications (ICAECC). IEEE, 2018.

20. Saeed, Mohammed, et al. "MIMIC II: a massive temporal ICU patient database to support research in intelligent patient monitoring." Computers in cardiology. IEEE, 2002.

21. Maryem, Baya, et al. "Diabetic patients and physicians' acceptability of a mobile health application for Diabetes monitoring in Fez region (Morocco)." 2020 1st International Conference on Innovative Research in Applied Science, Engineering and Technology (IRASET). IEEE, 2020.

22. Jeffree, M. S., Ahmedy, F., Avoi, R., Ibrahim, M. Y., Rahim, S. S. S. A., Hayati, F., ... \& Tuah, N. M. (2020). "Integrating Digital Health for Healthcare Transformation Conceptual Model of Smart Healthcare for northern Borneo." International Journal of Advanced Trends in Computer Science and Engineering, 9(1), 110-115.

23. Asaf-Ud-Doulah, Mohammed, et al. "Design and Implementation of A Wearable Monitoring System for Alzheimer's Patient and Elders." American Journal of Science \& Engineering 1.3 (2020): 40-44.

24. Freitas, Charles NC, Filipe R. Cordeiro, and Valmir Macario. "MyFood: A Food Segmentation and
Classification System to Aid Nutritional Monitoring." 2020 33rd SIBGRAPI Conference on Graphics, Patterns and Images (SIBGRAPI). IEEE, 2020. 